\title{
Proper motion of the spectrally selected structures in Herbig-Haro flows
}

\author{
T.A. Movsessian *and T.Yu. Magakyan ${ }^{\dagger}$ \\ Byurakan Astrophysical Observatory, Aragatsotn reg., Byurakan, 0213 Armenia
}

\begin{abstract}
We present some results of the investigations of proper motions of spectrally separated structures in the Herbig-Haro (HH) outflows with the aid of Fabry-Perot scanning interferometry. This project was started more than twenty years ago on the $2.6 \mathrm{~m}$ telescope of Byurakan Observatory and afterwards was continued on $6 \mathrm{~m}$ telescope (Russia). We describe the progress of research in the frames of this project and focus in more detail on the results of the latest observations of $\mathrm{HH} 83$ outflow system.

The method of Fabry-Perot scanning interferometry allowed to reveal the morphology of high and low velocity structures not only inside the terminal working surfaces of the jets but also in their internal knots. As the great advance we consider the development of a methodology for measuring proper motions of already separated kinematical structures using observations in two epochs, which made it possible to reveal the physical nature of these structures.

Concerning the HH 83 collimated outflow it should be noted that our first observations of HH 83 revealed two structures in its working surface with strongly different radial velocities, and the second epoch data allowed to measure their proper motions. The proper motions of these structures are nearly equal, which points that they are physically connected and represents forward and reverse shock regions in the terminal working surface of $\mathrm{HH} 83$.
\end{abstract}

Keywords: Star formation, Herbig-Haro objects, jets and outflows

\section{Introduction and previous achievements}

High proper motions (PM) of Herbig-Haro (HH) objects were discovered about 40 years ago. Orientation of PM vectors of $\mathrm{HH}$ objects, which represent shocked excitation regions, revealed the bipolar nature of high velocity flows, responsible for their formation (Herbig \& Jones, 1981, Jones \& Herbig, 1982). Further discoveries of highly collimated jets from young stellar objects indicated that $\mathrm{HH}$ objects actually are the brightest parts of these flows (Reipurth et al., 1986, 1993); in subsequent studies they usually are generically called $\mathrm{HH}$ jets and flows.

Further investigations of HH flows revealed their complex morphology. In particular, high and low excitation zones were separated by ground based and HST narrow band imagery (Hartigan et al., 2011, Reipurth et al., 1997) as well as by long-slit spectroscopy (Hartigan et al., 2011, Heathcote \& Reipurth, 1992, Reipurth et al., 1997). High and low velocity components in the terminal working surfaces (WS) and inside the certain knots in the jets were found. By the methods of spectra-imagery full morphology and kinematics of these structures was revealed. Low radial velocity components appear as bow shape structures, while in high velocities the compact knots are detected (Hartigan et al., 2000, Movsessian et al. , 2000, Movsessian et al., 2009).

To clarify the physical nature of these two kinematical structures we decided to measure proper motions of these structures using two epoch of observations with scanning Fabry-Perot interferometer (FPI). Already the first attempt was very successful: two epoch study of HL Tau jet, based on the observations in 2001 and 2007, allowed to estimate the PM of spectrally separated structures in the jet. This method, probably for the first time applied to $\mathrm{HH}$ jets, demonstrated that the structural components inside the jet, divided by low and high radial velocity, have, nevertheless, very similar values of PM (Movsessian et al., 2012). This

\footnotetext{
*tigmov@bao.sci.am, Corresponding author

†tigmag@sci.am
} 
important result was confirmed also for the components inside the FS Tau B jet system (Movsessian et al., 2019).

Generally speaking, inside the terminal WS (this is the common term for the regions, where supersonic flow slams directly into the undisturbed ambient medium: Reipurth \& Bally 2001), as well as in the internal WS of jets knots, two principal shock structures form. They include a 'reverse shock', which decelerates the supersonic flow, and a 'forward shock', which accelerates the material with which it collides (e.g. Hartigan, 1989).

HH 83 represents a wiggling knotty jet emerging from IRAS 05311-0631 source. This source, being deeply embedded in a molecular cloud, is not visible in optical range, but it illuminates edges of a conical cavity, formed by the outflow (Reipurth, 1989). At the distance of $105 \operatorname{arcsec}$ from the beginning of the jet, a bow shock structure was found, which is visible in $\mathrm{H} \alpha$ emission, but not in [SII] lines (Reipurth, 1989).

Our first observations of HH 83 with FPI revealed the separated structures with strongly different radial velocities in the terminal working surface (more than $250 \mathrm{~km} \mathrm{~s}^{-1}$ ), as well as confirmed the steady increase of velocities along the jet depending on the distance from the source (Movsessian et al., 2009).

HH 83 system represented itself the suitable object for the second epoch spectra-imagery observations, especially in view of discovery of two separated kinematical structures in its WS. However, HH 83 is much more distant object than, for example, Taurus dark cloud. Taking this into account we decided to enlarge the time between two epochs up to 15 years.

\section{Observations and Data Reduction}

Observations were carried out in the prime focus of the $6 \mathrm{~m}$ telescope of Special Astrophysical Observatory of the Russian Academy of Sciences in two epoches: 10 Feb. 2002 and 2 Feb. 2017, in good atmpospheric conditions (seeing was about 1 arcsec). We used a FPI placed in the collimatedl beam of the SCORPIO (Afanasiev \& Moiseev, 2005) and SCORPIO-2 (Afanasiev \& Moiseev, 2011) multi-mode focal reducers in 2002 and 2017, respectively. The capabilities of these devices in the scanning FPI observational mode are presented by Moiseev $(2002,2015)$. Description of the first epoch observations was presented in Movsessian et al. (2009).

During the second epoch observations the detector was a EEV 40-90 $2 \times 4.5 \mathrm{~K} \mathrm{CCD}$ array. The observations were performed with $4 \times 4$ pixel binning, so for each spectral channel $512 \times 512$ pixel images were obtained. The field of view was 6.1 arcmin with a scale of 0.72 arcsec per pixel. Second epoch observations provided more deep images and higher spectral resolution.

The scanning interferometer was ICOS FPI operating in the 751 st order of interference at the $\mathrm{H} \alpha$ wavelength, providing spectral resolution of FWHM $\approx 0.4 \AA$ (or $\approx 20 \mathrm{~km} \mathrm{~s}^{-1}$ ) for a range of $\Delta \lambda=8.7 \AA$ (or $\approx 390 \mathrm{~km} \mathrm{~s}^{-1}$ ) free from order overlapping. The number of spectral channels was 40 and the size of a single channel was $\Delta \lambda \approx 0.22 \AA\left(\approx 10 \mathrm{~km} \mathrm{~s}^{-1}\right)$.

In both epochs an interference filter with $\mathrm{FWHM} \approx 15 \AA$ centered on the $\mathrm{H} \alpha$ line was used for premonochromatization.

We reduced our interferometric observations using the software developed at the SAO (Moiseev, 2002, 2015, Moiseev \& Egorov, 2008) and the ADHOC software package ${ }^{1}$. After primary data reduction, subtraction of night-sky lines, and wavelength calibration, the observational material represents "data cubes". We applied optimal data filtering, which included Gaussian smoothing over the spectral coordinate with FWHM $=1.5$ channels and spatial smoothing by two-dimensional Gaussian with FWHM $=2-3$ pixels. The FPIs, used in two epochs of observations, had different spectral resolutions. Therefore the rebinned data cubes were created for both epochs to bring them into the same velocity steps. It allows to achieve the better comparison of morphological details, which have same radial velocities.

Using these data cubes, we spectrally separated the details with different radial velocity in the outflow system. Then PM were measured for the selected structures using observations in both epochs.

\section{Results}

During the observations the field of view of the SCORPIO focal reducer covered the entire HH 83 outflow system including the jet, the working surface and the reflection nebula around the source (which actually

\footnotetext{
${ }^{1}$ The ADHOC software package was developed by J. Boulestex (Marseilles Observatory) and is publicly available in the Internet.
} 
Table 1. Proper motions and radial velocities of knots in the HH 83 outflow

\begin{tabular}{lccccc}
\hline \hline Knot & $\begin{array}{c}\text { Distance } \\
(\operatorname{arcsec})\end{array}$ & $\begin{array}{c}\left.\mathrm{V}_{\text {tan }^{a}}{ }^{-1} \mathrm{~km} \mathrm{~s}^{-1}\right) \\
(\mathrm{deg})\end{array}$ & $\begin{array}{c}\mathrm{PA} \\
\left(\mathrm{km} \mathrm{s}^{-1}\right)\end{array}$ & $\begin{array}{c}\mathrm{V}_{a b s} \\
\left(\mathrm{~km} \mathrm{~s}^{-1}\right)\end{array}$ \\
\hline $\mathrm{D}$ & 13.5 & $120 \pm 30$ & 310 & -130 & 177 \\
$\mathrm{~F}$ & 19.3 & $142 \pm 35$ & 327 & -140 & 200 \\
$\mathrm{G}$ & 27.8 & $135 \pm 40$ & 319 & -165 & 213 \\
$\mathrm{WS}_{\text {high vel }}$ & 117.0 & $160 \pm 23$ & 282 & -312 & 351 \\
$\mathrm{WS}_{\text {low vel }}$ & 119.4 & $145 \pm 30$ & 277 & -50 & $153^{b}$ \\
counter bow & 96 & $180 \pm 45$ & 135 & +180 & 255 \\
\hline
\end{tabular}

(a) Tangential velocities correspond to $450 \mathrm{pc}$ as a distance of the flow

(b) Values of these velocities will be discussed below

represents the illuminated cavity walls). Below we will discuss all these parts of HH 83 separately and compare the observations of 2002 and 2017.

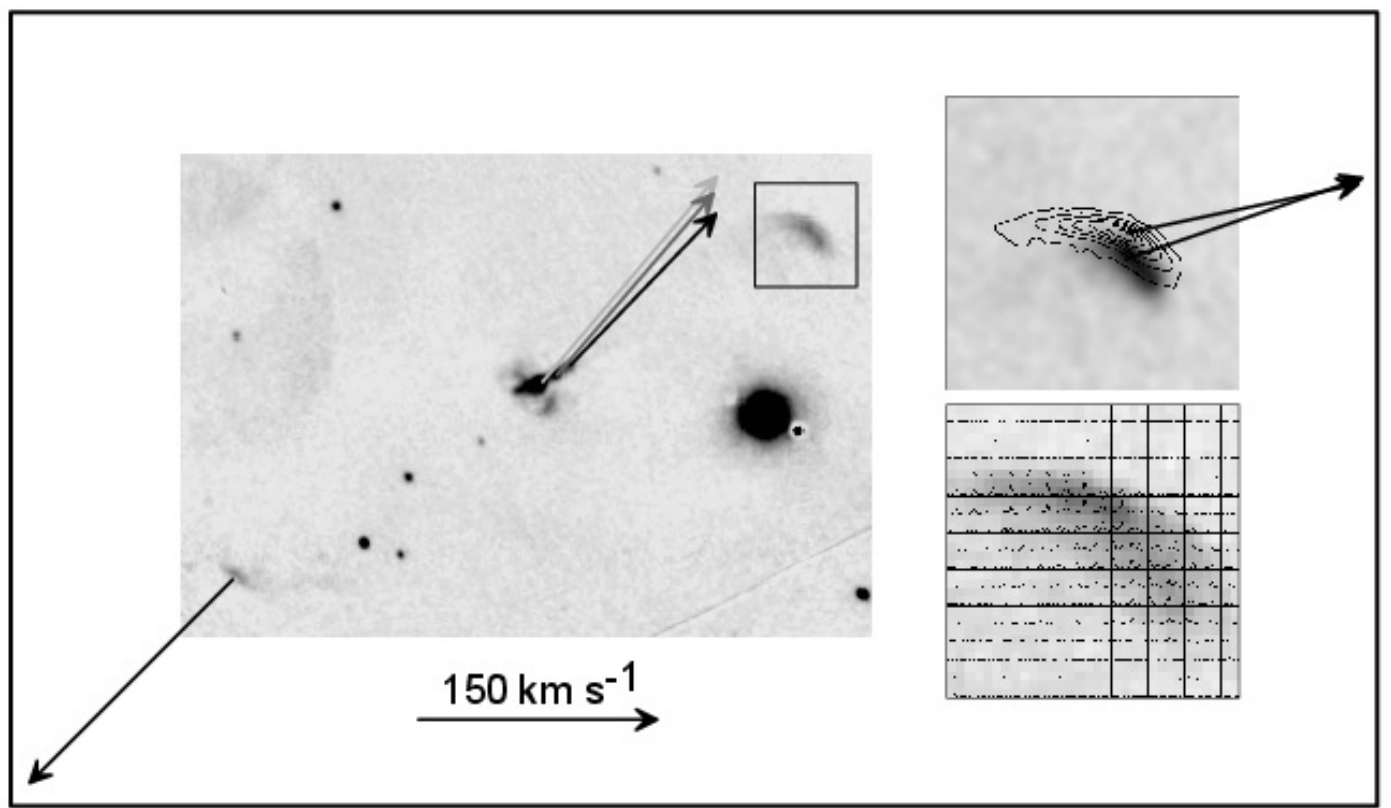

Figure 1. On the left panel the integrated over all velocity channels image of HH 83 outflow system with overlapped PM vectors in the jet knots and counterflow is presented. On the right bottom panel the grid of $\mathrm{H} \alpha$ profiles in the working surface, which indicates significant split of profiles in high and low velocity structures, is shown. On the right top panel the integrated over gaussian fitted profiles of low velocity component (grey scale) and high velocity one (contours) with PM vectors are presented.

\subsection{The terminal working surfaces, their proper motions and kinematical structures}

Besides of the already known WS (further denoted as NW-bow) which lies in about 2 arcmin distance from the source, our image clearly shows the second bow-shape structure in the opposite direction from the source, not described previously (Fig. 1, left panel). This structure lies on the axis of outflow system in the nearly same distance from the source as a main NW-bow. After re-examining of the first data, we found its faint traces in the same place. In contrast with the NW-bow this bow-shock has the positive radial velocity. It undoubtedly represents the terminal WS of the counter flow (further SE-bow), confirming the bipolar and symmetric nature of this outflow system.

As was already mentioned above, our previous data demonstrated that NW-bow in the HH 83 system is divided into two distinct components, which are well separated spectrally as well as spatially (see Fig. 6 in Movsessian et al., 2009). After gaussian fitting of the both components of $\mathrm{H} \alpha$ emission we restored images 
of these components for each observational epoch. Using these images we measured the PM of high and low radial velocity structures in $\mathrm{NW}$-bow.

The results of our PM measurements are presented on Fig. 1. In particular, PM vectors for the both low and high radial velocity structures of NW-bow of HH83 flow are shown in the inset. It is obvious that both structural components have very similar values of proper motion, despite the large difference in the radial velocities. We also estimated the distance between these structures during the second epoch observations and it turned out to be the same as for the first epoch (1000 AU). This is an additional evidence of their equal PM values. We will discuss this result in more detail in the next section.

On same Fig. 1 we show the PM vectors for the three brightest knots in the HH 83 jet, for the separated structures in the NW-bow as well as for the SE-bow, i.e. for the counter bow-shock. It worth to mention that this bow-shock has nearly the same range of radial velocities (in absolute values) as NW-bow, but, unlike the latter, it does not show neither spectral nor spatial inner separation. In any case, we were able to measure PM of this structure, even though it was significantly fainter in the first epoch data.

The numerical results are given in Table 1 where the distances for each structure, measured from the source position, values of tangential velocities (computed for the distance of $450 \mathrm{pc}$ ), position angles (PA) of PM vectors, radial velocities as well as the calculated absolute spatial velocities are presented.

\section{2. $\mathrm{PM}$ of the knots in the jet}

We measured PM of several bright knots in the HH 83 jet, namely knots D, G and F (according to the nomenclature of Reipurth, 1989). These knots also show high values of PM (see Table 1), similar to $\mathrm{PM}$ of high and low velocity structures in the terminal WS. It should be noted, however, that although the geometric axis of the jet coincides with NW-bow, the position angles of PM vectors of the jet knot significantly differ from those for the structures in NW-bow. Actually they are turned in the northern direction for about 14 degrees. Spatial velocities of the knots, calculated from their radial and tangential velocities, increase along with the distance from the source. Same trend also is observed in their radial velocities (see Table 1 ). We discuss this effect in the next section.

The knots in the HH 83 jet did not demonstrate the distinct division to high and low radial velocity components; consequently it was impossible to reveal the morphological structures with different radial velocities inside them.

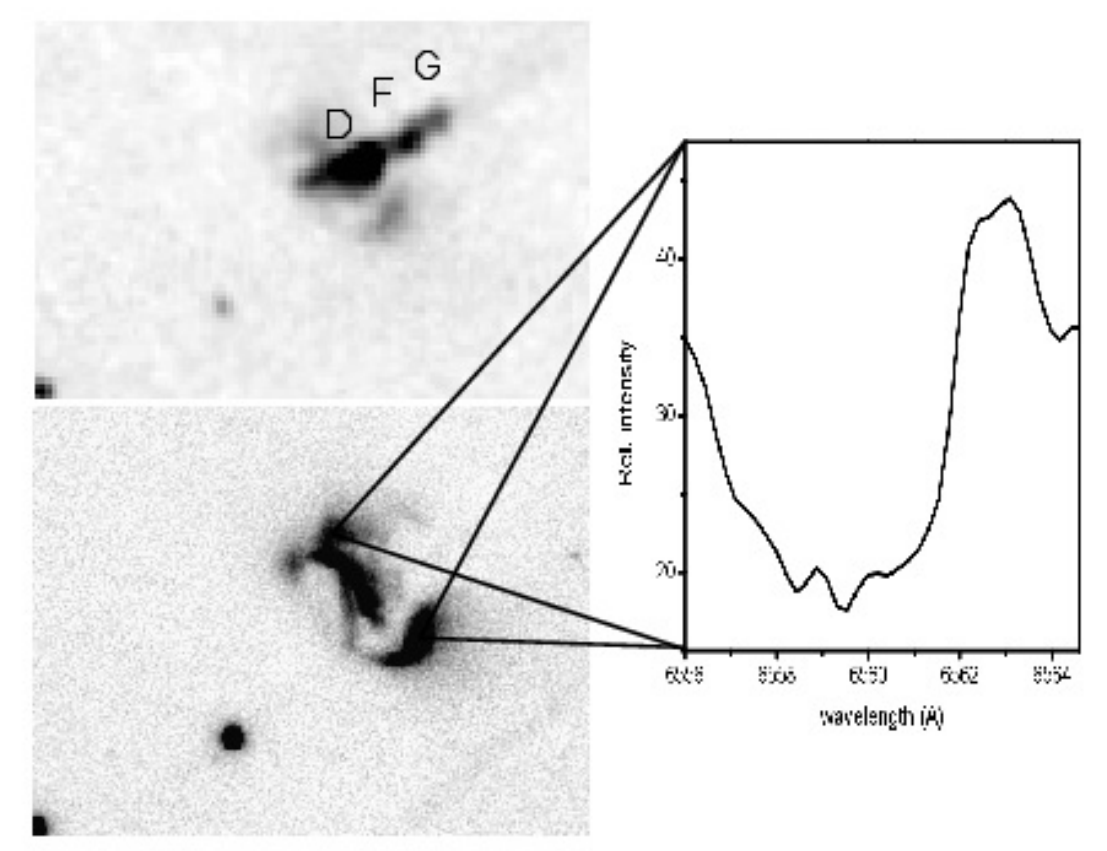

Figure 2. Integrated profile of the $\mathrm{H} \alpha$ line in the reflected light of the source of $\mathrm{HH} 83$. In the left side the enlarged image of the central part of HH 83 system is shown in $\mathrm{H} \alpha$ emission (the integrated FP image from 2017, upper panel) and in $i$ band (PanSTARRS survey, lower panel) 


\subsection{The reflection nebula}

Observations show that $\mathrm{HH} 83$ jet is propagating through the evacuated conical cavity, formed by wideangle wind from the deeply embedded infrared source (Reipurth et al., 2000). The walls of this cavity are illuminated by light from this source. In the optical range they form two reflection lobes around the narrow jet. In the high-resolution infrared images (Reipurth et al., 2000) one can get impression of several spiral or helical arms on the cone walls. On the PanSTARRS $i$ band image (see Fig. 2, lower panel) these two lobes are connected with bar-like structure, which probably represents one of the above mentioned arms. On the restored $\mathrm{H} \alpha$ image (Fig. 2, upper panel) these details are practically invisible, which confirms their reflection nature.

Above mentioned high quality of the new data allows the attempt to obtain the profile of $\mathrm{H} \alpha$ line in the spectrum of the central star through the scattered light of the source, deeply embedded in the dark cloud.To study the profile of $\mathrm{H} \alpha$ line in the reflected light we used the advantages of spectra-imagery, which allows to summarize data and to construct the total line profiles for any selected area. To avoid the contamination from the jet radiation and to deal with pure continual (i.e. reflected) light, we integrated profiles from the two side lobes only, excluding the bar-like structure, which is superimposed on the D knot of the jet. Resulting integrated $\mathrm{H} \alpha$ profile is presented in the right side of Fig. 2. This profile has strong and wide $\left(\sim 300 \mathrm{~km} \mathrm{~s}^{-1}\right)$ blue-shifted absorption with weak emission component near the zero radial velocity. We assume that it corresponds to the invisible IR source.

\section{Discussion and conclusion}

\subsection{Working surface}

Comparing the inner structures in the WS of HH 83 and in the knots in the HL Tau jet, we see that in both cases two emission structures with strongly different radial velocities were discovered. However, in the HL Tau jet these structures differ not only by their radial velocities but also by excitation, while in the parts of HH 83 WS the excitation level does not change, and these structures are visible mainly in $\mathrm{H} \alpha$ (Reipurth, 1989).

Their radial velocities differ in more than $250 \mathrm{~km} \mathrm{~s}^{-1}$. This is an unique case: for example, two velocity components in the WS of HH 111 differ for about $60 \mathrm{~km} \mathrm{~s}^{-1}$ only (Reipurth et al., 1997) and in the HL Tau jet - about $100 \mathrm{~km} \mathrm{~s}^{-1}$. This large difference in speed cannot be explained by existence of two separate outbursts, which formed two bow-shape structures with strongly different radial velocities, because in this case the high velocity structure will catch up the low velocity one in 15 years; besides, our new observations reveal that proper motions of these structures are the same.

Thus, we can come to conclusion that we observe in the terminal working surface of HH 83 physically connected structures, which can be designated as 'reverse shock' and 'forward shock'. The observed large difference in the radial velocities of these structures can form in very extremal conditions. For example, in the case of HL Tau jet the bright low velocity structures in front of the high velocity knots appear only in the region where the collisional interaction between the jet and wide outflow from XZ Tau is taking place (Movsessian et al., 2007).

In the case of HH 83 the asymmetry of bow-shock as well as the turning of it PM vectors compared to that of jet knots are the further indications of the encounter between the jet and the dense cloud core.

Taking into account the simple bow-shock and Mach disk model of Hartigan (1989), such values of velocities difference could occur when the ratio of ambient medium to jet densities will be near 100, and with the jet velocity more than $300 \mathrm{~km} \mathrm{~s}^{-1}$.

If our conclusion is right and the high velocity structure in the WS indeed represents 'reverse shock', than we detect emission of the decelerated flow matter; the ratio of pattern speed to flow speed in this structure by definition will be equal one. On the contrary, for the 'forward shock', which represents the accelerated matter of ambient medium, this ratio will be much higher due to the lower radial velocities of the emitting particles. The spatial velocities of the flow structures, presented in the Table 1, correspond to the moving matter, while in the case of the forward shock this value does not have physical meaning, being strongly dependent on the ambient medium conditions. On the other hand, since the velocity in the 'reverse shock' region corresponds to the real matter movement, it is possible to compute the inclination angle between the line of sight and the jet, which turns out to be near 27 degrees. Using all these values, one can estimate the kinematical age of the outflow about 1500 year. 


\subsection{The source IRAS $05311-0631$}

The analysis of the reflected spectrum of the HH 83 source shows that it has $\mathrm{H} \alpha$ line profile of $\mathrm{P}$ Cyg type, with a wide, nearly rectangular absorption and faint secondary blue-shifted peak. Such profiles are likely to be formed in a strong wind with optical depth sufficient to produce the deep blueshifted absorption component (e.g. Muzerolle et al., 2011). They are very typical for the FU Ori type eruptive stars; on the other hand, only a few T Tauri stars show such well developed P Cygni profiles at $\mathrm{H} \alpha$ line. The blue edge of the absorption trough indicates wind velocities of up to $300 \mathrm{~km} \mathrm{~s}^{-1}$. This value is nearly the same as the velocity of powerful wind that emanates from FU Orionis (e.g. Herbig et al., 2003).

As another argument for the FU Ori-type nature of IRAS 05311-0631 the detection of faint CO absorption bands at $2.3 \mu \mathrm{m}$ by Davis et al. (2011) can be considered. Such absorption bands, though usually more strong, are typical for FUors (e.g. Reipurth \& Aspin, 1997) .

On the other hand, FU Ori phenomenon in nearly all cases is associated with HH outflows (Audard et al., 2014). Moreover, the spacing of structure details in some HH outflows corresponds to the statistical estimates of the supposed recurrence of the FUor events (Herbig et al., 2003). 2003). In this case, the bright WS of HH 83 flow could be result of FU Ori-type outburst, which took place about 1500 year ago, judging from kinematical age of the outflow.

This work was supported by the Science Committee of RA, in the frames of the research project ${ }^{1} 18 \mathrm{~T}$ 1C329. Observations with the SAO RAS telescopes are supported by the Ministry of Science and Higher Education of the Russian Federation (including agreement No05.619.21.0016, project ID RFMEFI61919X0016).

\section{References}

Afanasiev V.L., \& Moiseev A.V., 2005, Astronomy Letters, 31, 194

Afanasiev V.L., \& Moiseev A.V., 2011, Baltic Astronomy, 20, 363

Audard, M., Ábrahám, P., Dunham, M.M. et al. 2014, in Protostars and Planets VI, Univ. Arizona Press, eds. H. Beuther et al., 387

Davis, C.J., Cervantes, B.,Nisini, B. et al., 2011, Astron. Astrophys. , 528, A3

Hartigan, P., 1989, ApJ, 339, 987

Hartigan, P., Morse, J., Palunas, P., et al. 2000, Astron. J. , 119, 1872

Hartigan, P., Frank, A., Foster, J. M., et al. 2011, Astrophys. J. , 736, 29

Heathcote, S. \& Reipurth, B. 1992, Astron. J. , 104, 2193

Herbig, G. H., \& Jones, B. F. 1981, Astron. J. , 86, 1232

Herbig, G. H., Petrov, P. P., \& Duemmler, R. 2003, Astrophys. J. , 595, 384

Jones, B. F., \& Herbig, G. H. 1982, Astron. J. , 87, 1223

Moiseev, A.V., 2002, Bull. Spec. Astrophys. Obs., 54, 74

Moiseev, A.V., 2015, Bull. Spec. Astrophys. Obs.,70, 94

Moiseev, A.V., \& Egorov, O.V. 2008, Bull. Spec. Astrophys. Obs., 63, 181

Movsessian, T. A., Magakian, T. Y., Amram, P., et al. 2000, Astron. Astrophys. , 364, 293

Movsessian, T. A., Magakian, T. Y., Bally, J., et al. 2007, Astron. Astrophys. , 470, 605

Movsessian, T. A., Magakian, T. Yu., Moiseev, A. V., \& Smith, M. D., 2009, Astron. Astrophys. , 508, 773

Movsessian, T. A., Magakian, T. Y., \& Moiseev, A. V. 2012, Astron. Astrophys., 541, A16

Movsessian, T. A., Magakian, T. Y., Burenkov, A. N. 2019, Astron. Astrophys., 827, A94.

Muzerolle, J., Calvet, N., Hartmann, L. 2001, Astrophys. J. , 550, 944. 
Reipurth, B., Bally, J., Graham, J. A., et al. 1986, Astron. Astrophys. , 164, 51

Reipurth, B., 1989, A\&A 220, 249

Reipurth, B. 1989, European Southern Observatory Conference and Workshop Proceedings, 247

Reipurth, B., Raga, A. C., \& Heathcote, S. 1996, Astron. Astrophys. , 311, 989

Reipurth, B., Raga, A. C., \& Heathcote, S. 1992, Astrophys. J. , 392, 145

Reipurth, B., \& Heathcote, S. 1992, Astron. Astrophys. , 257, 693

Reipurth, B., Heathcote, S., Roth, M., et al. 1993, Astrophys. J. Lett. , 408, L49

Reipurth, B., Hartigan, P., Heathcote, S., Morse, J., \& Bally, J. 1997, Astron. J. , 114, 757

Reipurth, B., Hartigan, P., Heathcote, S., et al. 1997, Astron. J. , 114, 757

Reipurth, B., \& Aspin, C.A. 1997, Astron. J. , 114, 2700

Reipurth, B., Yu, K. C., Heathcote, S., et al. 2000, Astron. J. , 120, 1449

Reipurth, B., \& Bally, J. 2001, Ann. Rev. Astron. Astrophys. , 39, 403 